\title{
The Shape Factor in Radial Keratotomy
}

\author{
P. A. J. STARR
}

London

\begin{abstract}
Summary
The corneal contour as defined by the shape factor was found by photokeratoscopy both before and after radial keratotomy to reduce myopia. In 51 eyes with mean pre-operative myopia of $-5.67 \mathrm{DS}$ there was a mean reduction of $-5.23 \mathrm{DS}$, and the shape factor became negative in all cases. A relationship was found between the pre-operative myopia and the shape factor, and the size of optical zone to be left untouched when attempting to achieve emmetropia. The importance of taking shape factor into account when forming the surgical plan was made apparent by retrospective study of the surgery performed.
\end{abstract}

Radial keratotomy is at present the most widely practised of the techniques of refractive corneal surgery. Epikeratoplasty, thermokeratoplasty, compression sutures, wedge resections and relaxing incisions, lathing of the frozen or non-frozen cornea and intra corneal lenses are other techniques currently being used to make patients less dependent on optical appliances. In all the procedures there is room for improvement in the accurate correction of refraction, and in radial keratotomy consideration of the corneal contour can make predictability more exact.

In 1969 Yenaliev $^{1}$ of the USSR introduced anterior radial keratotomy using freehand multiple incisions outside the physiological optical zone. He obtained a mean reduction of myopia by some four dioptres in 425 eyes. From 1972 onwards Fyodorov and Durnev carried out animal experiments and put the procedure on a less empirical basis, regulating the size of the untouched central area of the cornea or optical zone after taking into account the radii of curvature of the central cornea, the corneal diameter, and a factor relating to the elasticity of the corneal structure called scleral rigidity and measured by
Maklakov tonometry. The diameter of the central optical zone ${ }^{(d)}$ was found from the formula:

$$
d=\sqrt{10 R}, 3\left(12 R^{2}+A\right)-96 R^{2}-3 A
$$

when $\mathrm{R}=$ radius of curvature of the cornea

$\mathrm{D}=$ diameter of the cornea

$$
A=\left(D^{2}+\frac{16}{3}\left(R-\sqrt{\left.R-\frac{D^{2}}{4}\right)^{2}}\right)\left(\frac{k}{p+k \alpha}\right)^{2}\right.
$$

and $p=$ the required effect of keratotomy

$\mathrm{k}=$ rigidity coefficient of the cornea

$\alpha=$ the practical coefficient for the surgeon

By varying the size of the optical zone $(\mathrm{OZ})$ and the depth and number of incisions they were able to achieve a consistent reduction in myopia with an acceptable degree of predictability.

Bores $^{3}$ took the technique to the United States in 1978 and interest generated a new range of instruments in particular the micrometer-set guarded diamond blade and the ultrasound pachymeter. The Rusian work was repeated and it became clearer that there are a number of important variables and while 
Maklakov tonometry was less useful, a search continues for an alternative method of measuring corneal elasticity.

Diet $z^{4}$ has shown that the size of the optical clear zone selected by the surgeon is the single most important factor determining refraction change in radial keratotomy. By regression analysis he showed that 28 per cent of the variability in refractive outcome at one year after operation could be explained by difference in optical zone size.

\section{Shape Factor}

The shape of a high percentage of human corneas is close to an ellipsoid and in Euclidian geometry it can be described as a conic section. A conic section needs two parameters for its definition, the central curvature which defines size but is independent of shape, and the shape factor which is independent of size. Shape factor is a designation of the rate of flattening of the corneal periphery and is a convenient term to describe the profile of a cornea in one meridian. The traditional well accepted term for describing shape is eccentricity where

$$
\operatorname{Eccentricity~}(e)=\sqrt{1+\frac{b^{2}}{a^{2}}},
$$

$a$ and $b$ being the major and minor axes of the ellipse. However, it has been found more convenient to use the term shape factor which is simply the square of the eccentricity. Corneas with high shape factors have high rates of flattening and those with negative shape factors actually steepen in the periphery.

In 1970 Townsley $^{5}$ reported, for the first time, the distribution of the shapes of human corneas in a group of 350 patients. He showed that the peak of the distribution curve was at 0.25 but that some corneas were in the range 0.65 or greater while others had a negative shape factor. Patients with Keratoconus typically have high shape factors and the measurement is of considerable consequence in contact lens fitting. With radial keratotomy it is found that the prolate (pointed) side of the ellipse becomes flattened while the oblate (flatter) side is steepened. Since a large decrease in shape factor accompanies success- ful radial keratotomy it was thought worthwhile to study the changes brought about by this surgery.

\section{Computer Assisted Photokeratoscopy}

Historically corneal curvature has usually been measured with an ophthalmometer/keratometer type of instrument. The underlying principle of the keratometer assumes that the surface being measured is spherical but with a cornea this is rarely the case. Also the instrument measures radius of curvature by finding the chord length between two reflection points and since the cornea is aspheric the central curvature calculated from a chord of approximately $3 \mathrm{~mm}$ cannot define the corneal profile. Attempts to perform paracentral keratometry readings are restricted by difficulties in controlling a patient's fixation, and the angle between the visual axis and the points of reflection.

Goode $^{6}$ first invented what we now know as the Placido disc, and the photokeratoscope made by Placido ${ }^{7.8}$ was subsequently popularised by Gullstrand. Beginning in 1966 when Ludlam and Wittenberg ${ }^{9.10}$ published a series of papers and defined three criteria; firstly, that the image plane of the photokeratoscope which is virtual and behind the cornea should be flat. In order to achieve a flat image plane the target surface should be ellipsoidal. The target system in the Wesley Jessen Photoelectric Keratometer consists of seven concentric rings which form an ellipsoidal surface so that when reflected in a $44.00 \mathrm{D}$ cornea the flat virtual image is of 7 rings, the smallest being $3 \mathrm{~mm}$ in diameter and increasing in steps of $1 \mathrm{~mm}$ to the outermost which is $9 \mathrm{~mm}$ in diameter.

Secondly, there must be accurate reproducible alignment of the patient's visual axis with that of the instrument. For accuracy the Wesley Jessen PEK Mark IIIA has a very small depth of field of $0.1 \mathrm{~mm}$. Alignment is obtained by a cross wire placed at the principle focus of the instrument so that to the patient it appears at infinity. Reproducibility is achieved by allowing the observer to ensure that the incident light is normal to the corneal surface at the moment of taking a photograph.

Thirdly, they stated that data analysis should be rigorous, detailed and accurate. 
Table I The diameter of the optical zone in millimetres selected to achieve emmetropia when 8 radial incisions are made at 95 per cent Paracentral Pachymetric Depth (Neumann)

\begin{tabular}{cccccccc}
\hline$K$ & $1.50-2.0$ & $2.25-3.0$ & $3.25-4.0$ & $4.25-5.0$ & $5.25-6.0$ & $6.25-7.0$ & $7.25-8.0$ \\
\hline $41.00-42.00$ & 4.0 & 3.50 & 3.0 & 2.75 & & & \\
$42.24-44.00$ & 4.25 & 3.75 & 3.25 & 3.0 & 2.75 & & \\
$44.25-46.00$ & 4.50 & 4.0 & 3.50 & 3.25 & 3.0 & 2.75 & \\
$46.25-48.00$ & 5.0 & 4.50 & 4.0 & 3.50 & 3.25 & 3.0 & 2.75 \\
\hline
\end{tabular}

Presentation of the shape of the cornea in three dimensions is difficult and System 2000 analyses the photographs in two principle meridians only making a more manageable two dimensionsal presentation as commonly considered by ophthalmologists. Each meridian shows the fourteen reflection points corresponding with the seven target rings. Optical ray tracing principles are used and the information is computed in terms of sagittal depths and semi-chord lengths.

\section{Patients and Methods}

Fifty-one eyes of 34 patients were examined before and after radial keratotomy. The age range was from 22 to 57 years and the pre-operative degree of myopia from $-3.25 \mathrm{DS}$ to $-10.50 \mathrm{DS}$. The mean being $-5.67 \mathrm{DS}$ spherical equivalent. Pre-operative astigmatism of 0.0 to $1.75 \mathrm{D}$. Cyl. was present but in this series no attempt was made surgically to correct it.

If hard contact lenses were used they were not worn for two weeks prior to examination, and if soft they were removed for one week. Corrected visual acuity was recorded and cycloplegic refraction was carried out. Ocular dominance for distance was determined by fixing a distant object through a $2 \mathrm{~cm}$ square in a card held at arms length. The eyes were carefully examined for ocular pathology. Corneal shape was analysed from a polaroid photograph using the Wesley Jessen Photoelectric Keratometer Mark IIIA. Analysis of the keratographs was made at the Wesley Jessen Computer Centre, Cobham, Surrey, and the results presented in the System 2000 form used for contact lens design. Sixteen of the eyes also had keratometry and a record of shape factor by the Humphrey Autokeratometer. Measurements of corneal thickness were made using a DGH Pachymeter immediately before surgery. The operation plan was drawn up after reference to Table I (Neumann ${ }^{11}$ ).

Following the instillation of one drop of Benoxi- nate the centre of the optical zone was marked in the epithelium of the cornea with the flat side of the tip of a 20-gauge needle while the patient looked at the centre of the filament of a Zeiss Mark 6 operating microscope. ${ }^{12}$ All surgery was under general anaesthesia and the optical zone and radial lines were marked with light impression and Brilliant Green. A diamond blade micrometer-set guarded KOI Knife was used after the blade extension had been checked against a coin guage. Eight incisions were made from the limbus to the optical zone at a single depth setting of 95 per cent of the lowest paracentral reading and no stepped or re-deepening incisions were used. Wound irrigation was by balanced salt solution through a Rycroft canula and Chloramphenicol drops were instilled at the end of the procedure. Tulle gras, a pad and bandage were applied overnight and the eye was uncovered the next morning. FML Neo drops were instilled three times daily for 10 days.

Post-operative Polaroid photographs were not taken until good sharp ring reflections could be obtained, and in general this was between 6 and 12 months after operation.

\section{Results}

The mean pre-operative myopia of $-5.67 \mathrm{DS}$ was reduced by -5.23 DS to a mean of -0.44 DS. The mean pre-operative shape factor taken as an average of the vertical and horizontal measurements was reduced by 0.7 from +0.245 to -0.46 . In order to seek a relationship between pre-operative myopia and shape factor, and the optical zone size that achieves close to zero post-operative error, the results were treated as follows:

Those intances where the residual refractive error was not one dioptre or less were eliminated, and the remaining 45 eyes were regarded as being successful at the chosen optical zone size. Assuming the relationship between the optical zone and the other variables is of the form 
$\mathrm{OZ}=\mathrm{a}+\mathrm{b}_{1}($ Myopia Pre-op) $)+\mathrm{b}_{2}($ Shape $)+\mathrm{b}_{3}$ (Average a regression analysis gave the following results Standard

Variable Par

Error of

Myopia

pre-op

Shape

$\begin{array}{lcc}\text { Average } \mathrm{K} & \mathrm{b}_{3} & 0.0098 \\ * * & \text { highly significant, } & \text { p-value }<0.001\end{array}$

t-value 0.458

0.0125

0.140

0.0108

$6.75 *$

$-3.87^{* *}$

0.90
The estimate of the parameter $b_{3}$ for the average $K$ variable is not significantly different from zero and there is no evidence that the average $K$ value is related to the optical zone, when the pre-operative myopia and shape factor are taken into account. $\mathrm{K}$ is therefore omitted from the regression, and the parameters are estimated in

$\mathrm{OZ}=\mathrm{a}+\mathrm{b}_{1}$ (Myopia pre-op) $+\mathrm{b}_{2}$ (Shape)

Standard

Error of

Variable Parameter Estimate Estimate t-value

$\begin{array}{lllll} & \text { a } & 3.813 & 0.072 & 52.9^{* *}\end{array}$

$\begin{array}{lllll}\text { pre-op } & b_{1} & 0.082 & 0.0123 & 6.7^{* *}\end{array}$

$\begin{array}{lllll}\text { Shape } & b_{2} & -0.519 & 0.137 & -3.8^{* *}\end{array}$

** highly significant, p-value $<0.001$

Taking the relationship between optical zone and myopic pre-op and shape factor we have

$$
\begin{gathered}
\mathrm{OZ}=3.813+0.082 \text { (Myopia pre-op) } \\
-0.519 \text { (Shape) }
\end{gathered}
$$

and any values of myopia and shape can be entered in the above equation to give an optical zone, which can be rounded to the nearest $0.25 \mathrm{~mm}$ to give the optical zone to be used.

Confirmation was obtained by taking from Table II the 45 cases regarded as successful at the chosen optical zone size and grouping them by zone size employed. The mean preoperative status of all the cases in each optical zone size group, in terms of myopic error and shape factor, was calculated as a paired comparison using the formula

$$
\begin{aligned}
& \text { Mean } x=\frac{1}{n} \sum_{i=1}^{n} x 1 \\
& \text { Mean } y=\frac{1}{n} \sum_{i=1}^{n} x i
\end{aligned}
$$

Where $\mathrm{n}$ is the total number of cases

$\mathrm{i}$ is $1,2, \ldots \ldots \mathrm{n}$

$\mathrm{x}$ is the shape factor

$\mathrm{y}$ is pre-operative myopic error

The outcome is a mean point for each opti- cal zone size representing the average of the coordinates of all the cases in that group. The means are plotted in Figure 1 and are seen to be very close to a straight line having their origin near to zero on both axes. The slope suggests that the original myopic error is the most important factor affecting the choice of zone size, but that shape factor is also a major contributor.

On Figure 1 there are superimposed circular quadrants which represent the hypothetical limits of the correction which can be achieved with the technique described and given bands of myopia and shape factor.

\section{Discussion}

The reduction in shape factor brought about by Radial Keratotomy was described by Henslee and Rowsey ${ }^{13}$ in 1983 but they were unable to correlate the power of the pre-operative shape factor with the change in cycloplegic refraction. They believed that shape factor did not allow the prediction of the potential refractive change with Radial Keratotomy, but it is to be noted that they used the Humphrey Keratometer which measures horizontal chord lengths $13.5^{\circ}$ nasally and temporally from the visual axis; a unique curve fit algorithm of the aplanatic corneal surface is analysed to designate a shape factor. In the present study 16 of the eyes had comparisons of the Wesley Jessen PEK and Humphrey shape factors, and no satisfactory correlation could be found between the two sets of measurements. This was true, even when the horizontal Humphrey shape factor was compared with that of the Wesley Jessen apparatus except in so far as a more negative shape factor resulted, in keeping with the change from a parabola to a trapezoid shape.

Various nomograms have been produced to determine operative parameters and have been derived from the clinical experience of the surgeon. Rowsey ${ }^{14}$ used a very simple method to attempt to correct the myopia. With a blade setting of 90 per cent of the central pachymetry reading he used a $3.0 \mathrm{~mm}$ marking trephine for myopia of $-4.0 \mathrm{D}$ or more, a $3.5 \mathrm{~mm}$ trephine in myopia of -3.0 to $-3.75 \mathrm{D}$, and a $4.0 \mathrm{~mm}$ trephine for myopia of -2.0 to $-2.75 \mathrm{D}$ spherical equivalent. 
Table II Findings in 51 cases undergoing radial keratotomy

\begin{tabular}{|c|c|c|c|c|c|}
\hline Name & $\begin{array}{l}\text { Myopia } \\
\text { pre-op }\end{array}$ & $\begin{array}{l}\text { Myopia } \\
\text { post-op }\end{array}$ & $\begin{array}{l}\text { Average } K \\
\text { reading }\end{array}$ & $\begin{array}{l}\text { Optical } \\
\text { zone }\end{array}$ & $\begin{array}{c}\text { Shape factor } \\
\text { pre-op }\end{array}$ \\
\hline M.A. & -10.50 & -3.50 & 44.25 & 3.00 & $+0.40^{*}$ \\
\hline M.A. & -8.50 & -0.75 & 44.43 & 3.00 & +0.17 \\
\hline \multirow[t]{2}{*}{ M.B. } & -5.50 & 0.00 & 44.18 & 3.25 & +0.32 \\
\hline & -4.50 & +0.75 & 44.18 & 3.25 & +0.32 \\
\hline \multirow[t]{2}{*}{ D.B. } & -3.75 & -0.25 & 43.37 & 3.25 & +0.24 \\
\hline & -3.50 & +0.75 & 43.12 & 3.25 & +0.18 \\
\hline R.B. & -4.00 & +0.50 & 42.24 & 3.25 & +0.14 \\
\hline \multirow[t]{2}{*}{ B.D. } & -4.75 & 0.00 & 44.68 & 3.25 & +0.44 \\
\hline & -4.75 & -0.25 & 44.31 & 3.25 & +0.37 \\
\hline \multirow[t]{2}{*}{ V.D. } & -4.50 & 0.00 & 40.75 & 3.25 & +0.22 \\
\hline & -5.25 & +0.25 & 41.50 & 3.00 & +0.37 \\
\hline \multirow[t]{2}{*}{ J.F. } & -7.75 & +0.25 & 42.93 & 3.00 & +0.19 \\
\hline & -9.50 & -2.50 & 43.62 & 3.00 & $+0.05^{*}$ \\
\hline \multirow[t]{2}{*}{ A.G. } & -6.75 & 0.00 & 45.18 & 3.00 & +0.44 \\
\hline & -5.75 & 0.00 & 45.56 & 3.25 & +0.37 \\
\hline D.H. & -5.75 & +0.75 & 44.68 & 3.25 & +0.37 \\
\hline \multirow[t]{2}{*}{ S.H. } & -6.50 & 0.00 & 42.50 & 3.25 & +0.13 \\
\hline & -6.00 & +0.75 & 42.99 & 3.25 & +0.20 \\
\hline H.L. & -4.00 & -0.25 & 43.37 & 3.25 & +0.28 \\
\hline M.M. & -3.00 & +0.25 & 44.43 & 3.75 & +0.15 \\
\hline \multirow[t]{2}{*}{ A.T. } & -7.75 & -3.25 & 41.93 & 3.00 & $+0.36^{*}$ \\
\hline & -7.50 & -0.75 & 41.99 & 3.00 & +0.39 \\
\hline R.B. & -4.15 & +0.50 & 43.18 & 3.25 & +0.27 \\
\hline \multirow[t]{2}{*}{ K.M. } & -6.00 & 0.00 & 46.68 & 3.00 & +0.48 \\
\hline & -6.00 & -0.75 & 46.37 & 3.00 & +0.57 \\
\hline \multirow[t]{2}{*}{ M.M. } & -4.00 & 0.00 & 46.18 & 3.50 & +0.27 \\
\hline & -4.25 & +0.25 & 46.37 & 3.50 & +0.18 \\
\hline \multirow[t]{2}{*}{ R.W. } & -3.75 & -0.25 & 42.14 & 3.25 & +0.17 \\
\hline & -3.50 & 0.00 & 42.62 & 3.25 & +0.41 \\
\hline \multirow[t]{2}{*}{ H.B. } & -5.50 & -0.50 & 43.25 & 3.25 & +0.22 \\
\hline & -5.75 & 0.00 & 43.37 & 3.25 & +0.14 \\
\hline A.B. & -7.50 & -0.50 & 44.43 & 3.00 & +0.50 \\
\hline \multirow[t]{2}{*}{ R.L. } & -4.50 & 0.00 & 42.81 & 3.50 & +0.25 \\
\hline & -6.00 & 0.00 & 43.37 & 3.50 & +0.07 \\
\hline L.M. & -4.00 & -0.50 & 44.62 & 3.50 & +0.28 \\
\hline \multirow[t]{2}{*}{ D.W. } & -3.25 & +1.00 & 41.12 & 3.50 & +0.23 \\
\hline & -3.25 & +0.25 & 41.62 & 3.50 & +0.21 \\
\hline \multirow[t]{2}{*}{ A.Y. } & -4.00 & +0.50 & 41.65 & 3.25 & +0.59 \\
\hline & -3.50 & +0.25 & 41.40 & 3.25 & +0.31 \\
\hline \multirow[t]{2}{*}{ E.L. } & -6.50 & 0.00 & 44.56 & 3.00 & +0.11 \\
\hline & -7.00 & -1.00 & 44.74 & 3.00 & +0.34 \\
\hline J.B. & -5.75 & +0.25 & 43.47 & 3.25 & +0.10 \\
\hline E.E. & -4.75 & -0.75 & 44.75 & 3,25 & +0.15 \\
\hline C.K. & -4.75 & +1.00 & 45.75 & 3.25 & +0.32 \\
\hline J.M. & -6.75 & 0.00 & 40.25 & 3.25 & +0.18 \\
\hline \multirow[t]{2}{*}{ A.O. } & -3.50 & 0.00 & 42.62 & 3.50 & +0.09 \\
\hline & -4.00 & 0.00 & 42.50 & 3.75 & +0.18 \\
\hline N.P. & -7.50 & -1.75 & 42.68 & 3.00 & $+0.34^{*}$ \\
\hline M.T. & -6.75 & -0.25 & 43.12 & 3.00 & +0.19 \\
\hline N.R. & -9.00 & -3.25 & 42.70 & 3.00 & $+0.28^{*}$ \\
\hline F.S. & -10.00 & -3.50 & 45.00 & 3.25 & $+0.44^{*}$ \\
\hline
\end{tabular}

* Not regarded as successful at chosen optical zone size.

Schachar ${ }^{15}$ produced a table to indicate the optical zone size necessary to correct a given refractive error depending on the keratometry.
Arrowsmith, Deitz and Sawelson ${ }^{16}$ emphasised age as a factor which affects the amount of correction achieved and their nomograms divide patients into those of 20 and 40 years of 


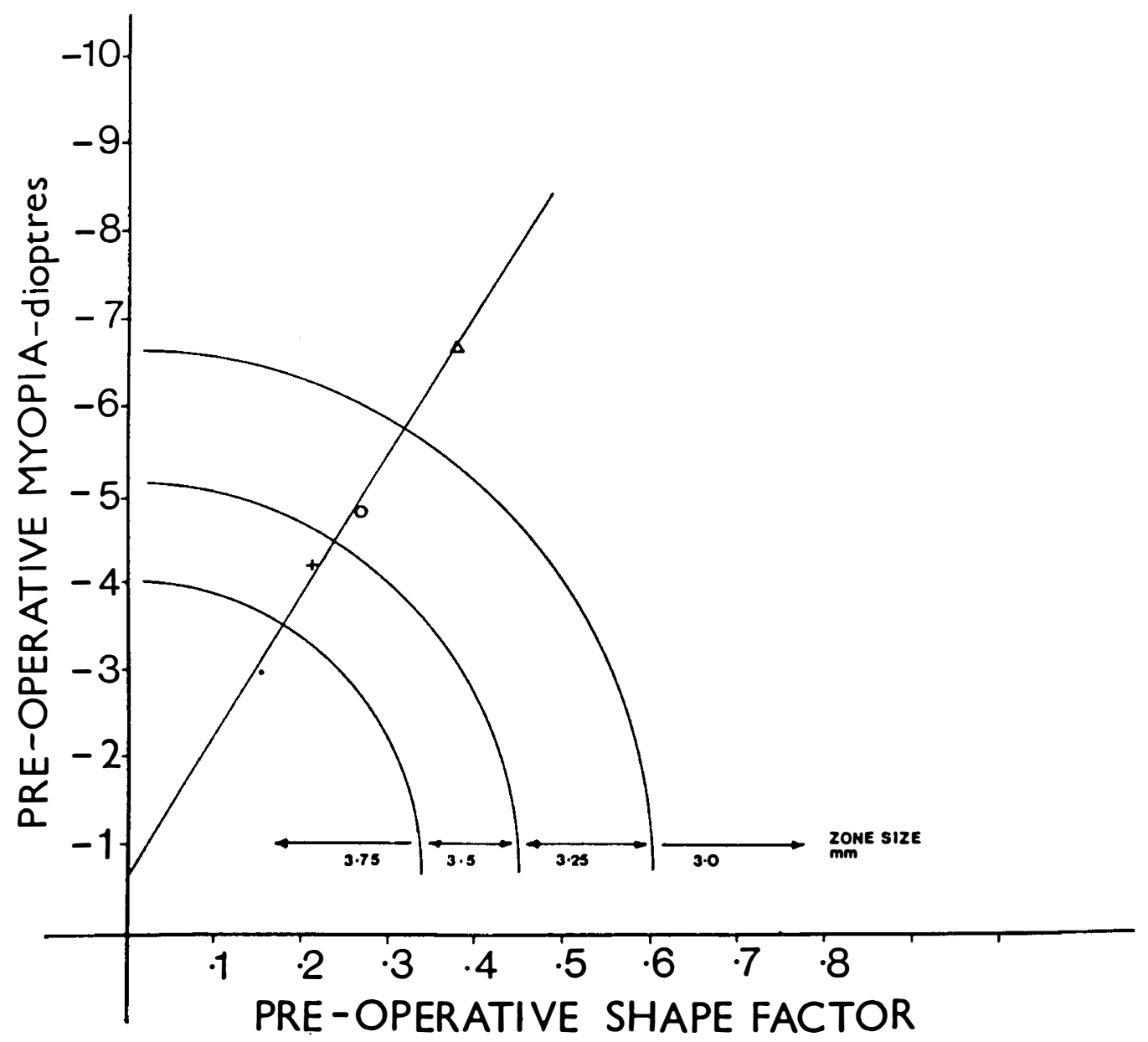

Fig. 1. Scale showing the optical zone size used to achieve a successful reduction of myopia.

age, the latter requiring larger optical zones or shallower incisions. Thornton has additional modifications for female eyes which act if they are younger than men's, and has a modifying schedule if intraocular pressure is above 19 $\mathrm{mmHg}$, or below $11 \mathrm{mmHg}$.

Fyodorov considers scleral rigidity measured by Maklakov Tonometry as being an important parameter and this leads to the need to introduce corneal diameter into the calculations. It is purported that the arrangement of collagen fibrils may be found to correlate with tissue elasticity and this could be determined by a paralimbal biopsy and electron microscopy. ${ }^{17}$ Waring, Lynn et al. ${ }^{18}$ used photokeratoscope pictures in the PERK study to measure changes in corneal topography but they found it a complex task to translate these measurements into clinically useful information and did not present their results.

Corneal shape factor is seen as a parameter which can improve the predictability of radial keratotomy. It is shown that the successful optical zone size relates far better to myopia and shape factor than to myopia and $\mathrm{K}$ reading. The slope of the line in Figure 1 suggests that the original myopic error is the most important factor affecting the choice of zone size, but the shape factor is also a major contributor.

The higher degrees of myopia with a low shape factor achieve a greater correction when an optical zone of a set size is used; conversely, the higher the shape factor, the less the reduction in myopia. Ignorance of the shape factor can allow an error of $0.75 \mathrm{~mm}$ or 
20 per cent in the size of the optical zone diameter, and variation from the arc of the appropriate zone size would result in under or overcorrections. Furthermore the graph predicts that in some eyes a reduction of -7.50 DS is possible using the technique described, while in eyes with high shape factors it is not.

Shape factor is an item which can be entered into computer programmes to assist the surgeon to plan his radial Keratotomy procedures, and has relevance for other modes of refractive surgery.

The author would like to thank the Department of Clinical Epidemiology of the Royal Free Hospital for statistical analysis and Mrs. Rachel Gubbins for compilation of figures and typing.

\section{References}

1 Yenaliev FS: Experience in surgical treatment of myopia. Vestn. Oftalmol. 1979; 3: 52-5.

${ }^{2}$ Fyodorov SN, Durnev VV: Operation of dosaged dissection of the corneal circular ligament. Ann. Ophthalmol. 1979; 11: 1885-90.

${ }^{3}$ Bores L: American experience with myopia procedure of Fyodorov, Keratorefraction (Ed. Schachar $R$ et al.) LAL Publishing. Denison, Texas. 1980; 175-81.

${ }^{4}$ Dietz MR et al.: Radial Keratotomy: An overview of the Kansas City Study presented at the 88th Annual Meeting of the A.A.O., Chicago, Illinois, October 1983. Ophthalmology 1984; 91: 5: 467-78.
5 Townsley M: New knowledge of the corneal contour Contacto 1970; 13 (3): 38-43.

${ }^{6}$ Goode H: Trans. Cambridge Phil. Soc. 1847; 8: 495

${ }^{7}$ Placido A: Novo instrumento de esploracae da cornea. Periodico Oftalmol. Practica. 1880; 5: 27-30.

× Placido A: Novo instrumento par alanyse immediate das irregularidades da cornea. Periodico Oftalmol. Practica. 1880; 6: 44-9.

${ }^{9}$ Ludlum W, Wittenberg S: Measurements of the ocular dioptic elements utilizing photographic methods. Am. J. Optom. 1966; 42: 249-67.

${ }^{10}$ Ludlum W, Wittenberg S, Rosenthal J, et al. : Photographic analysis of the ocular dioptic components. Am. J. Optom. 1967; 44: 276-96.

$"$ Neumann AC, Osher RH, Fenzl RE: Radial Keratotomy: A comprehensive evaluation. Doc ophthalmol. 1984;56: 275-301.

12 Steinberg EB, Waring GO: Comparison of two methods of marking the visual axis on the cornea during radial keratotomy. Am. J. Optom. 1983; 96: $605-8$.

${ }^{1.3}$ Henslee SL, Rowsey JJ: New corneal shapes in keratorefractive surgery. Ophthalmology 1983; 90:245-50.

1+ Rowsey JJ, Balyeat HD, et al.: Predicting the results of radial keratotomy. Ophthalmology 1983: 90: 642-54.

${ }^{15}$ Schachar RA, Black TD, Huang T: Understanding radial keratotomy. LAL Publishing, Denison, Texas. 1981; 277-284

16 Arrowsmith PN, Dietz MR, Marks RG, Sanders DR, Sawelson H: Radial keratotomy. SLACK Publishing, Thorofare, New Jersey. 1984.

${ }^{17}$ Fyodorov SM: London Symposium on Radial Keratotomy. Optician 1986; 191: 20-23.

18 Waring GO, Lynn MJ, et al.: Results of the Prospective Evaluation of Radial Keratotomy (PERK) Study one year after surgery. Ophthalmology 1985; 92: 177-99. 